\title{
Advancement of High Power Quasi-CW Laser Diode Arrays For Space-based Laser Instruments
}

\author{
Farzin Amzajerdian ${ }^{\mathrm{a}}$, Byron L. Meadows ${ }^{\mathrm{a}}$, Nathaniel R. Baker ${ }^{\mathrm{b}}$, Renee S. Baggott ${ }^{\mathrm{c}}$, \\ Upendra N. Singh ${ }^{a}$, Michael J. Kavaya ${ }^{a}$ \\ ${ }^{a}$ NASA Langley Research Center, MS 468, Hampton, Virginia 23681-2199 \\ ${ }^{b}$ Lockheed Martin Engineering and Science Company, Langley Research Center, MS 468, Hampton, \\ Virginia, 23681 \\ 'Science and Technology Corporation, Langley Research Center, MS 468, Hampton, Virginia, \\ 23681
}

\begin{abstract}
Space-based laser and lidar instruments play an important role in NASA's plans for meeting its objectives in both Earth Science and Space Exploration areas. Almost all the lidar instrument concepts being considered by NASA scientist utilize moderate to high power diode-pumped solid state lasers as their transmitter source. Perhaps the most critical component of any solid state laser system is its pump laser diode array which essentially dictates instrument efficiency, reliability and lifetime. For this reason, premature failures and rapid degradation of high power laser diode arrays that have been experienced by laser system designers are of major concern to NASA. This work addresses these reliability and lifetime issues by attempting to eliminate the causes of failures and developing methods for screening laser diode arrays and qualifying them for operation in space.
\end{abstract}

Keywords: laser diode array, laser diode pump, solid-state laser, lifetime, reliability, space-based laser instruments.

\section{INTRODUCTION}

The reliability of moderate to high power solid-state lasers that can operate autonomously over a sufficiently long period are constrained by their laser diode pump arrays (LDAs). For laser remote sensing instruments operating in space, the reliability and lifetime of LDAs are particularly critical because of high development and launch costs of instruments and their inaccessibility in space. Therefore, it has become imperative for NASA to consider LDAs as a major risk area for laser-based instruments when faced with limited commercial availability, limited reliability, and lack of statistical data required for screening and predicating their reliability. Subsequently, NASA has established a task to address the LDA issues under the Laser Risk Reduction Project. As part of this effort, a LDA characterization and lifetime test facility was developed at Langley Research Center to support several activities towards improving the reliability, lifetime, and efficiency of LDAs.

The leading causes of sudden failure and premature degradation of laser diode arrays are intrinsic semiconductor defects, optical facet breakdown resulting from excessive localized heating, and thermo-mechanical stresses due to the extreme thermal cycling of the laser active regions ${ }^{1-4}$. Experimental techniques and instrumentation have been developed to investigate these failure mechanisms and causes of premature degradation in order to guide the technology advancement, leading to highly reliable and very long lifetime laser diode arrays ${ }^{5}$. Several areas of improvement in the packaging and fabrication process of laser diodes have already been identified and efforts towards implementing these improvements are well underway. These efforts include the use of advanced high thermal conductivity materials for packaging of laser diode arrays and new fabrication techniques for mechanically attaching laser diode bars and submounts. 


\section{LDA Characteristics and Operations}

The solid state laser design and the characteristics of its lasing materials define the operating wavelength, pulse duration, and power required of the laser diode arrays. In general, the continuous-wave and high repetition rate $(>100 \mathrm{~Hz})$ pulsed solid state lasers require continuous-wave laser diodes either in a single element or 2-D array configuration. On the other hand, high pulse energy lasers operating at a relatively low repetition rate require quasi-CW 2-D array laser diodes. This work focuses on the latter, since most NASA laser applications require moderate to high power pulsed lasers and the fact that the reliability and lifetime issues associated with the quasi-CW LDAs are more serious. Table 1 summarizes the general quasi-CW LDA pump specifications and reflects the current state of the technology. These specifications are based on pump requirements for 1-micron and 2-micron solid state lasers that are the main laser sources meeting almost all NASA's remote sensing needs. The major differences between pump requirements for the Neodymium based 1micron lasers and the Thulium/Holmium-based 2-micron lasers are their operating wavelength and pulse duration. The 2-micron lasers require a pump wavelength of around 10 to $20 \mathrm{~nm}$ shorter compared with 1-micron lasers and a pump pulse duration of 5 to 10 times longer ${ }^{6,7}$. Table 1 provides the pump wavelengths and pulse durations for commonly-used Nd:YAG and Tm,Ho:YLF (pumped along c-axis) lasers.

TABLE 1. LDA parameters for pumping $1 \mu \mathrm{m}$ and $2 \mu \mathrm{m}$ solid state lasers.

\begin{tabular}{|l|c|c|}
\hline Parameter & 1-micron & 2-micron \\
\hline Central Wavelength (nm) & $808+/-2$ & $792+/-3$ \\
\hline Spectral Width (nm FWHM) & 2 & 3 \\
\hline Peak Power Per Bar (W) & 100 & 100 \\
\hline Pulse Width (msec) & 0.2 & 1.0 \\
\hline Duty Cycle & $2 \%$ & $2 \%$ \\
\hline Electrical Efficiency & $50 \%$ & $50 \%$ \\
\hline
\end{tabular}

The longer pump pulse duration required by 2-micron lasers causes the laser diode active material to experience a considerably larger temperature rise and more drastic thermal cycling. This translates to a shorter lifetime compared to the laser diodes used for 1-micron lasers. The level of thermal effects of longer pulse duration is illustrated in Figures 1 and 2 where an intensity decrease, wavelength shift and linewidth broadening are clearly evident. Figure 1 shows a substantial increase in linewidth when operating the LDA at pulse durations of $1 \mathrm{msec}$ and pulse repetition frequency (PRF) of $12 \mathrm{~Hz}$, as opposed to $200 \mu \mathrm{sec}, 60 \mathrm{~Hz}$. Though the duty factor of $1.2 \%$ is maintained for both cases, the line width increases from $2.1 \mathrm{~nm}$ to $3.8 \mathrm{~nm}$. The center wavelength shifts by about $1.5 \mathrm{~nm}$, which corresponds to a

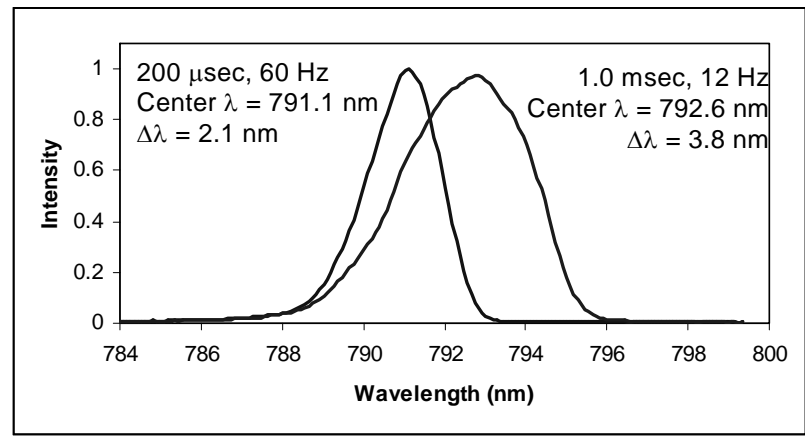

Figure 1. Spectrum of a LDA output operating at $200 \mu \mathrm{sec}$ and 1 msec pulse widths while maintaining $1.2 \%$ duty factor.

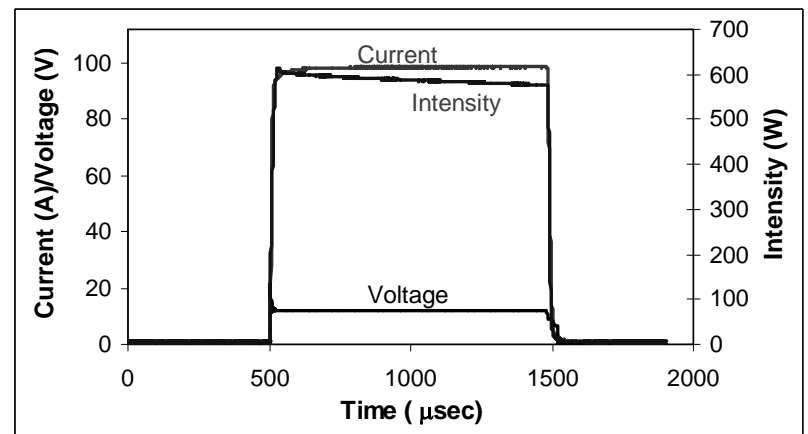

Figure2. Decrease in output power over a $1 \mathrm{msec}$ pulse duration.

temperature rise of about $6^{\circ} \mathrm{C}$. Figure 2 further illustrates the importance of thermal management for relatively long pulse durations ( $>200 \mu \mathrm{sec}$ ), as are needed for solid-state 2 micron laser systems. The output intensity decreases about $30 \mathrm{~W}$ over the 1 msec pulse duration resulting from localized heating of the LDA active region. This points to considerable pulse-to-pulse temperature cycling, which in turn accelerates the degradation of the LDA. Therefore, improving the efficiency of heat extraction from the active region is even more critical when the LDA are operated at longer pulse duration. 


\section{Characterization and Lifetime Testing}

Accurate characterization and lifetime testing under intended operating condition is a necessary step towards addressing the LDA reliability issues and developing proper space flight screening and qualification protocols. For this reason, a Laser Diode Characterization Facility (LDCF) has been developed for evaluating the performance, reliability, and lifetime of various LDAs under different set of operational conditions. The LDCF consists of two measurement stations: a Laser Diode Characterization Station and a Lifetime Test Station. The Characterization Station provides the basic characteristic parameters such as power, wavelength, linewidth, and efficiency. This setup is also capable of providing some specialized measurements that includes thermal profiling of laser diode facet and package, near and far field beam profiling, and high-resolution spectral measurements. The Lifetime Test Station, as illustrated in Fig. 3, is capable of simultaneous measurement of 12 LDAs using a common set of instruments for accurate comparative analysis and evaluation. The Lifetime Test Station is fully automated using a single computer to set operational and environmental parameters, acquire and archive data, flag anomalous data, and generate a number of warning and status alert messages when necessary.

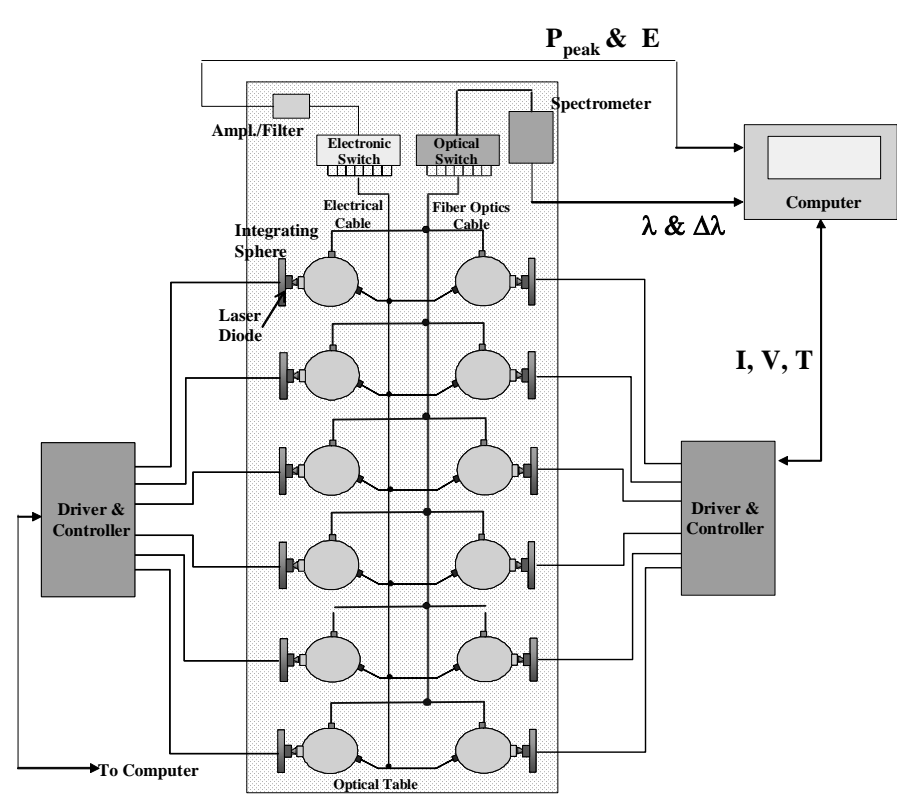

Figure 3. LDA Lifetime Test Setup.

temperature increase that occurs as a result of overall package heating (typically given as $\sim 0.25 \mathrm{~nm} /{ }^{\circ} \mathrm{C}$ ). Other effects can also be monitored such as line width broadening or decreased efficiency to measure junction temperature rise. The modern high resolution infrared cameras can provide complementary datas about the thermal properties of LDAs. A valuable IR imaging data is the measure of heat spread over the LDA facet. The IR camera being utilized here has a spatial resolution of 50 microns and its temperature sensitivity is $0.1^{\circ} \mathrm{C}$.

An example of this laser diode measurement is shown in Fig. 4 where the thermal profile of a LDA facet is measured before and after an anomalous event. The thermal images clearly show considerable temperature rise due to failure of a number emitters within the top bar. The generated excess heat expanding over the
As mentioned earlier, thermal characteristics of LDAs is a key factor affecting the reliability and lifetime of LDAs. Therefore particular attention was devoted in thermal measurements of LDAs. A prerequisite to determining the effects of junction temperature rise and thermal cycling on LDA lifetime and performance is to accurately measure these parameters under the intended operating conditions. Such measurements are also important for evaluating various packages for improved thermal properties. It is important to note that two separate thermal issues are involved. The first is removal of waste heat created by operation of the device; the second is the effect of the thermal cycling that takes place due to QCW operation.

Given the physical dimensions of an LDA, it is impractical to physically attach thermal probes sufficiently close to the active region for direct measurements. Thus, a common method for determining the junction temperature rise is to monitor secondary effects. One such technique involves monitoring the wavelength shift to diagnose junction

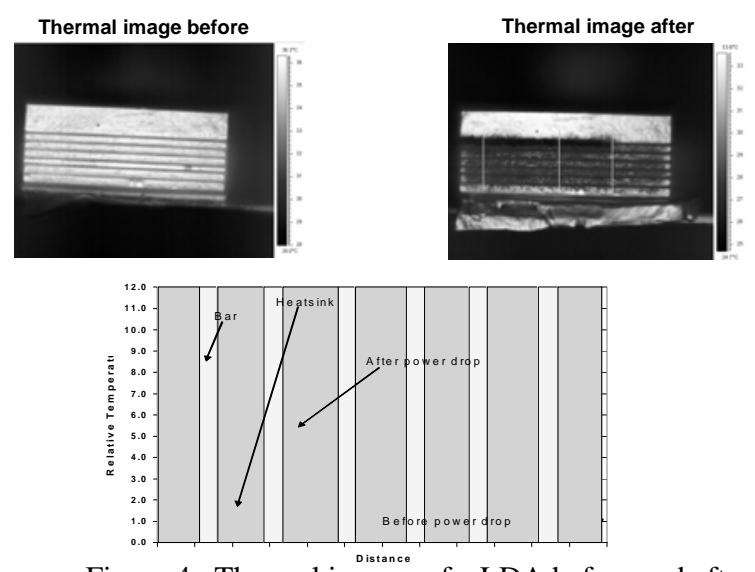

Figure 4. Thermal images of a LDA before and after an anomalous event. 
array can result in a chain reaction which causes first the failure of the remaining emitters within the same bar and then damaging the other bars. This progression of LDA failure is often observed in practice with different degree of severity and time scale.

The IR measurements are also effective in providing quantitative comparison of different packages and materials since the efficiency of heat extraction from the active regions of laser diode is the key to high reliability and long lifetime. Figure 5 is an example of the comparative measurements evaluating the performance of standard A and G package arrays produced by the same vendor. Figure 5 shows a fairly typical temperature distribution across a 6-bar array during operation as measured using the IR camera. The typical A-package bars operate over $4^{\circ} \mathrm{C}$ hotter than the typical G-package. Though not evident in Figure 5, which represents only one frame of data, additional analysis indicates slightly higher average temperature of the central bars of the A-package as compared to the G-package bars, which are generally cooler and more uniform in temperature. A-package diodes are cooled from one side producing a gradient across the bars whereas G-package diodes are cooled more uniformly from underneath at a consistent distance from the cold plate. For G-package arrays, this in turn equates to better output characteristics such as narrower spectral width, higher power and efficiency, and possibly longer lifetime.

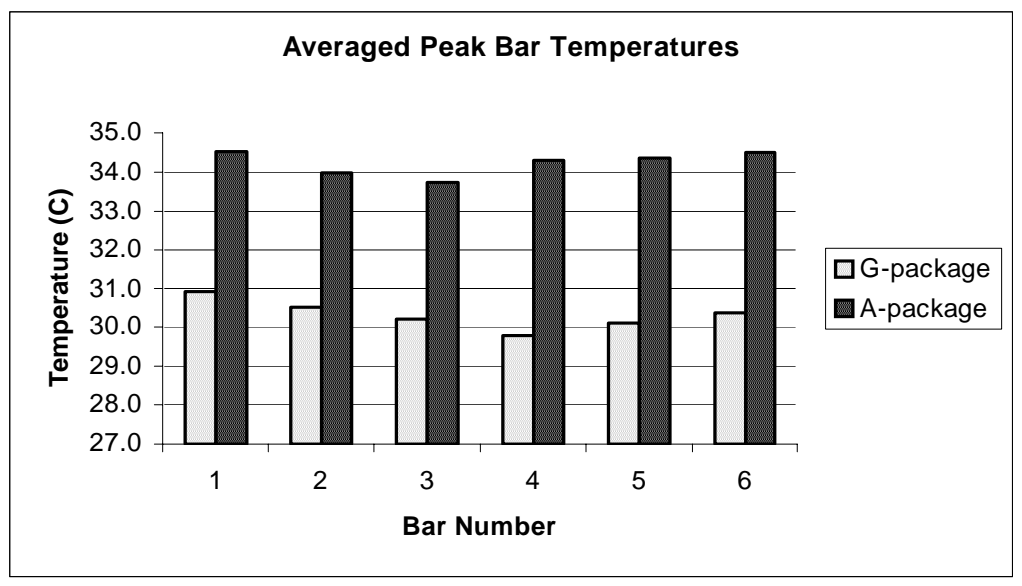

Figure 5. Thermal distribution across A and G-package LDAs.

\section{Advanced Packaging Technologies}

Since excessive heat and thermal cycling of the LDA active regions plays such a key role in limiting the reliability and lifetime of high power quasi-CW, particularly for the long pulsewidth operation, efforts are being made to improve the heat extraction efficiency. This is being done by utilizing advanced materials, for packaging LDAs, which have high thermal conductivity and a coefficient of thermal expansion (CTE) matching that of the laser bars. Figure 6 illustrates the major conductively-cooled package types currently being used by the LDA suppliers. The design of these packages needs to accommodate conducting a relatively high drive current through the bars and efficiently extract the excess heat from the bars, while limiting the mechanical stresses due to any CTE mismatch. The materials of choice for the LDA
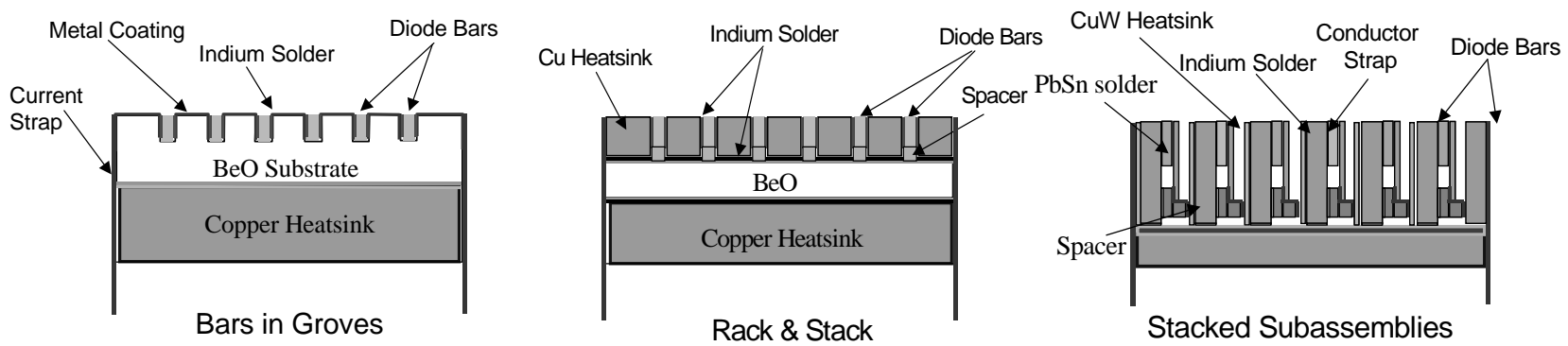

Figure 6. Different types of conductively-cooled, high power, LDA packages.

packages have been beryllium oxide, copper, and copper tungsten with indium solder as the bonding material.

Under the auspices of this effort, a number of advanced materials are being investigated that include CVD diamond, matrix metal composites, and carbon-carbon composites/graphite foam. As part of this effort, a custom-designed package housing six 100W bars using a CVD diamond material has already been fabricated by Northrop 
Grumman/Cutting Edge Optronics. The results of experimental evaluation of this package indicate substantial improvement in heat dissipation efficiency. Higher heat dissipation efficiency of the diamond packages is evident from Figure 7, which shows the diamond package cooling to a lower equilibrium temperature at a considerably faster rate than the $\mathrm{Cu} / \mathrm{BeO}$ package after the array is turned off. Figure 5 further shows the thermal cycling of the arrays in QCW operation at $10 \mathrm{~Hz}$. The amplitude of the thermal cycling is also expected to be lower even though it is not apparent from the temperature plots of Figure 7 due to spatial and temporal averaging of the images and the fact that they are not corrected for emissivity.

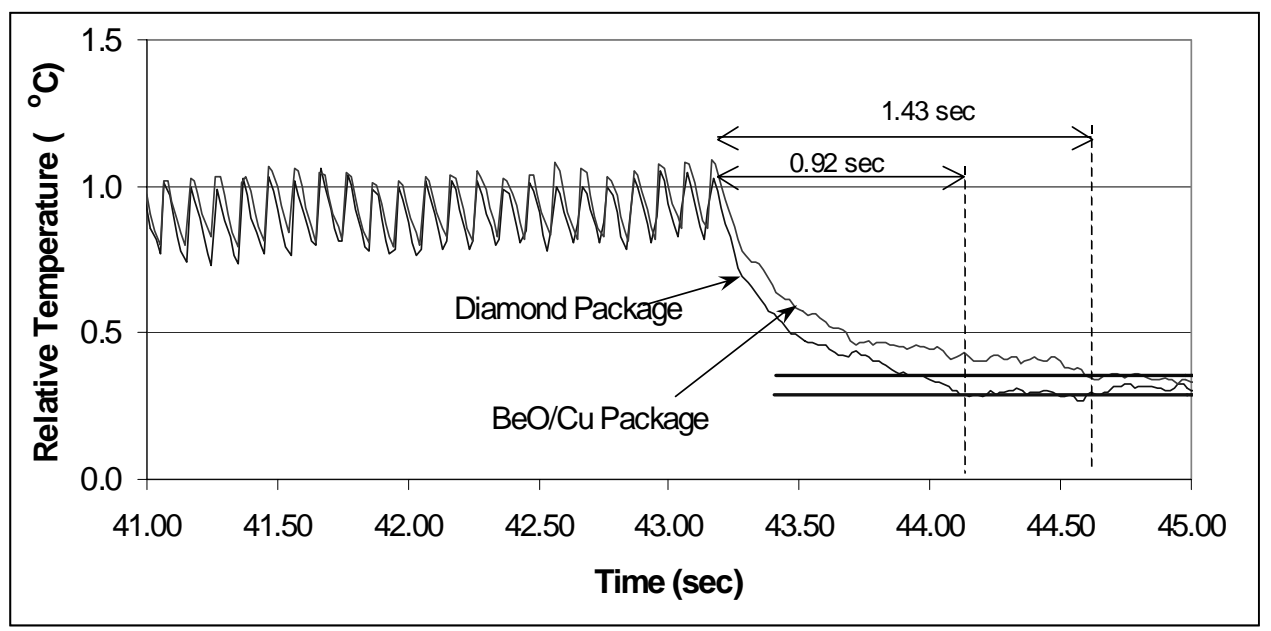

Figure 7. Thermal cycling and heat extraction efficiencies of Diamond and $\mathrm{Cu} / \mathrm{BeO} \mathrm{LDA}$ packages.

The heat rejection efficiency of this package was determined by running the array at a constant current and repetition rate of $80 \mathrm{~A}$ and $10 \mathrm{~Hz}$ respectively, while measuring its output wavelength and electrical efficiency at different pulsewidths. Fig. 8 is an example of the measurement results showing the laser diode temperature rise as a function of dissipated heat in the package for the diamond package and a similar package with $\mathrm{BeO}$ substrate and copper heatsink. The slope of the temperature vs. heat curve provides a figure of merit, referred to as thermal resistance, for each package's heat rejection efficiency. The measurements of Fig. 8 indicate a reduction of about $17 \%$ in thermal resistance of the diamond package. This is significant, as it can lead to a substantial increase in LDA lifetime. Using the classical Arrhenius relationship, typically applied to electronics, one could expect an increase in 'usable' life of roughly $20 \%$. However, the LDA lifetime may not be governed by the Arrhenius equation ${ }^{8,9}$ that is based on the active region temperature as the only stressor, in which

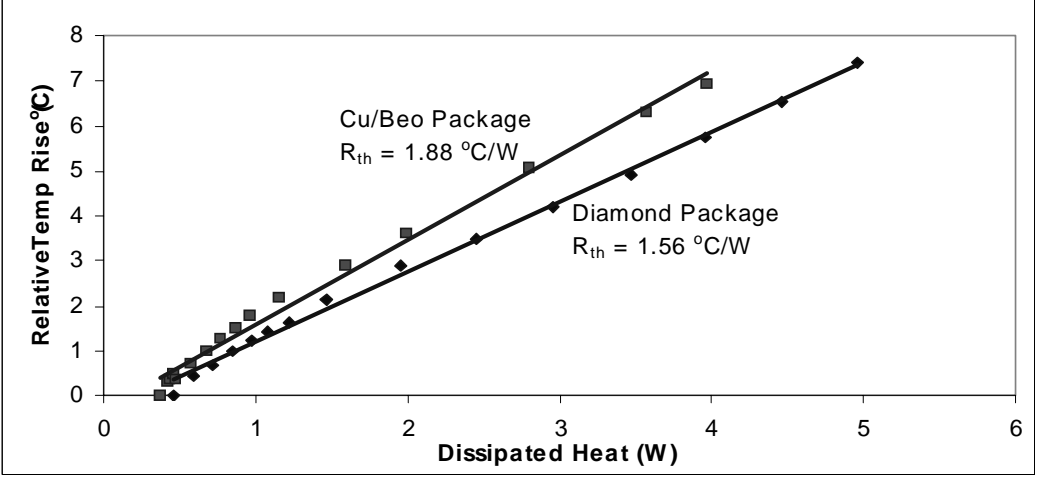

Figure 8. Thermal Characteristics of $600 \mathrm{~W}$ LDAs in diamond and $\mathrm{BeO} / \mathrm{Cu}$ packages. case a more complex reliability model is needed to better represent the LDA performance. This is the object of the lifetime testing effort that is currently underway. 


\section{ACKNOWLEDGMENTS}

The authors would like to express appreciation to NASA's Earth Science Technology Office and office of Exploration Systems for funding this effort through the Laser Risk Reduction Program.

\section{References}

1. B. Lu, E. Zucker, et al, "High power, high reliability CW and QCW operation of single AlGaAs laser diode array design,” SPIE Proc, 3945, 293-300, 2000.

2. W. C. Tang, et al, "Raman microprobe study of the time development of AlGaAs single quantum well laser facet temperature on route to catastrophic breakdown,” Appl. Phys. Lett., 58, 557-559, 1991.

3. H. C. Casey and M. B. Panish, Heterostucture lasers, Academic Press, NY, 1978.

4. N. I. Katsavets, et al, "Study of correlation between optical characteristics and mirror facet temperatures of the active region in high power SCH SQW InGaAsP/GaAs laser diodes,” SPIE Proc, 2148, 152-156, 1994.

5. Byron Meadows, Farzin Amzajerdian, Nathaniel Baker, Vikas Sudesh, Upendra N. Singh, and Michael J. Kavaya, “Thermal characteristics of high-power, long pulse width, quasi-CW laser diode arrays,” SPIE Proc, 5336, 203-211, 2004.

6. Norman P. Barnes, Mark Storm, Patricia Cross, and Milton Skplaut, "Efficiency of Nd laser materials with laser diode pumping,” IEEE J. of Quantum Elec., 26, 558-569, 1990.

7. Jirong Yu,, Alain Braud, and Mulegeta Petros, “600mJ, Double pulsed 2 micron laser,” Opt. Lett., 28, 540-542, 2003.

8. M. Fukuda, Reliability and degradation of semiconductor lasers and LEDs, Artech House Inc., MA, 1991.

9. F. Dorsch and F. Daiminger, “Aging test of high power diode lasers as a basis for an international lifetime standard," SPIE Proc, 2870, 381-389, 1996. 